\title{
Is phacoemulsification with viscosynechiolysis effective for angle closure glaucoma? Promising results from a pilot study
}

\author{
V Swetha E. Jeganathan ${ }^{1,2,3}$, Conrad Schmoll $^{3}$ and Pankaj K Agarwal ${ }^{3}$ \\ ${ }^{1}$ Department of Ophthalmology, University Hospital Ayr, Scotland, United Kingdom \\ ${ }^{2}$ University of Edinburgh, Scotland, United Kingdom \\ ${ }^{3}$ Princess Alexandra Eye Pavilion, Edinburgh, Scotland, United Kingdom
}

\begin{abstract}
Purpose: To determine the safety and efficacy of phacoemulsification and viscosynechiolysis (VSL) as a surgical management of angle closure with significant peripheral anterior synechiae (PAS).

Method: Retrospective series of patients with acute or chronic angle closure glaucoma with more than 90 degrees of PAS that were surgically treated with combined phacoemulsification and VSL. Patients were followed up over a two year period. Success was defined as intraocular pressure of $<21$ mmHg with or without glaucoma medications.

Results: Of 17 eyes in 12 patients, 5 had bilateral phacoemulsification with VSL. Two thirds were female, with a mean age of 75 years. Ten eyes had prior peripheral iridotomy. The mean pre-operative and post-operative IOP were 23.2 and $15.5 \mathrm{mmHg}$ respectively, with an average reduction of $8.4 \mathrm{mmHg}$. The median number of glaucoma medications decreased from 2 pre-surgery to 1 post-surgery. No eyes required further IOP lowering procedures.

Conclusion: VSL is an effective and safe procedure in controlling IOPs in patients with moderate to extensive PAS and could therefore delay or potentially prevent glaucoma filtration surgery.
\end{abstract}

\section{Introduction}

Primary angle closure glaucoma (PACG) is a leading cause of glaucoma blindness in the world. The World Health Organization estimates that by 2020, 5.3 million people with glaucoma will become blind from PACG [1]. In Scotland, PACG accounts for approximately $23 \%$ of newly diagnosed glaucoma cases [2]. The management of PACG becomes challenging when the trabecular meshwork is occluded by significant peripheral anterior synechiae (PAS). Persistently raised intraocular pressure (IOP) as a result of insufficient drainage from the trabecular meshwork then results in irreversible glaucomatous damage. Lens extraction which results in a significant IOP reduction, is now recommended both in the management of uncontrolled chronic PACG and as the initial intervention in acute PACG [3], but does not remove PAS. Combined phacoemulsification with goniosynechiolysis (p-GSL) has been advocated as a means of opening the angle with such patients, but the procedure is time consuming and prone to bleeding [4]. Other treatment interventions including trabeculectomy with or without lens extraction, glaucoma implants and cyclodestructive procedures also demonstrate poor success rates [5].

We describe a novel technique, phacoemulsification and viscosynechiolysis (p-VSL) (Figure 1) where following phacoemulsification, a viscoelastic agent is used to dissect the PAS and effectively widen the drainage angle (Figure 2). The aim of our pilot study is to determine the safety and efficacy of p-VSL as a surgical management of angle closure with moderate to extensive PAS. To the best of our knowledge, there have been no publications on this technique to date.

\section{Patients and methods}

We performed a prospective study at the Princess Alexandra Eye Pavillion in Edinburgh of 24 consecutive eyes from 19 patients with acute or chronic angle closure glaucoma with more than 90 degrees of PAS who were surgically treated with combined p-VSL. We used the following criteria to define angle closure glaucoma: presence of glaucomatous optic neuropathy with compatible visual field loss

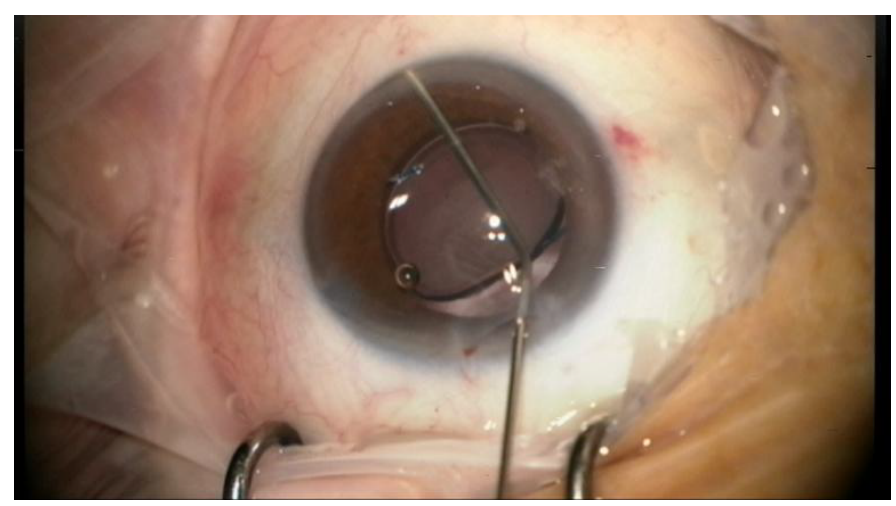

Figure 1. Phacoemulsification and viscosynechiolysis (p-VSL).

Correspondence to: Dr. V. Swetha E. Jeganathan, Department of Ophthalmology, University Hospital Ayr, Dalmellington Road, Ayr KA6 6DX, Scotland, United Kingdom; Email: vswetha@ausdoctors.net

Received: March 28, 2016; Accepted: April 19, 2016; Published: April 22, 2016 


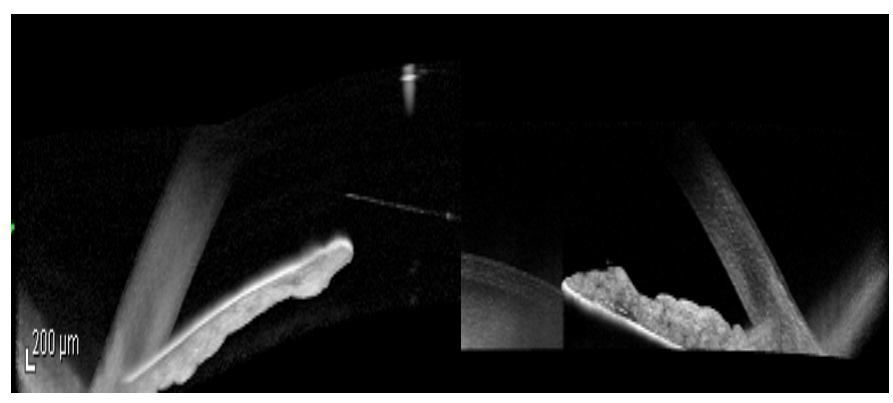

Figure 2. Anterior segment OCT demonstrating preop PAS and postop separation of the synechiae and widening of the anterior chamber angle following p-VSL.

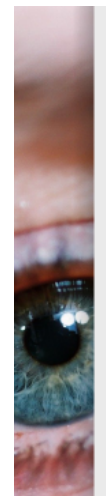

Ocular; clinical and demographic data

\begin{tabular}{|c|c|c|}
\hline \multicolumn{2}{|l|}{ No. Eyes } & 24 \\
\hline \multicolumn{2}{|l|}{ No. Patients } & 19 \\
\hline \multicolumn{2}{|l|}{ Age (years): } & Mean $75.66 \pm 6.69$ \\
\hline \multirow[t]{2}{*}{ Diagnosis: } & CACG & $91.3 \%$ \\
\hline & AACG & $13.0 \%$ \\
\hline \multicolumn{2}{|c|}{ Prior peripheral iridotomy: } & $75 \%$ \\
\hline \multicolumn{2}{|c|}{ Axial length (mm): } & Mean $22.05 \pm 0.95$ \\
\hline \multicolumn{2}{|c|}{ IOL power (Dioptres): } & Mean $22.69 \pm 8.67$ \\
\hline
\end{tabular}

Figure 3. The clinical characteristics of our patient population.

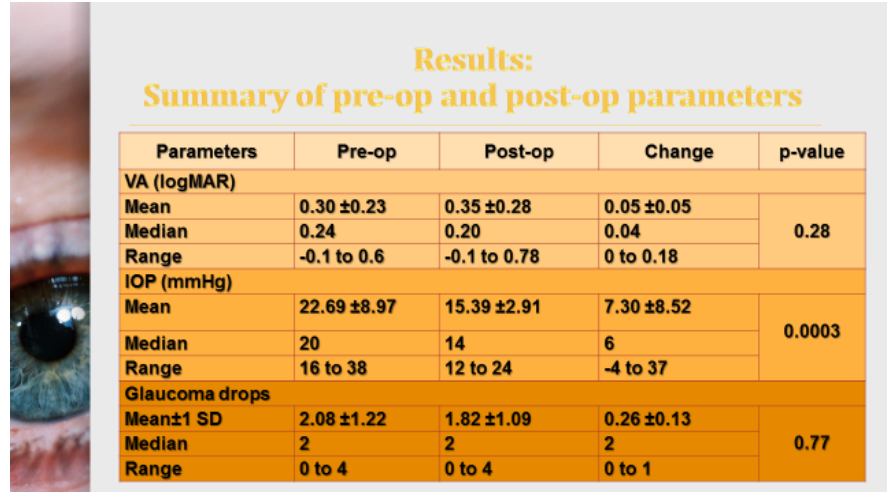

Figure 4. The summary of pre-operative and post-operative parameters.

in association with a closed angle on indentation gonioscopy. The study protocol adhered to the Tenets of the Declaration of Helsinki and informed consent was obtained from all subjects prior to commencement of p-VSL.

Our outcome measures included pre-operative and post-operative IOP, number of IOP lowering drugs, best corrected visual acuity (by Snellen chart, later converted to logarithm of minimum angle of resolution, $\log \mathrm{MAR}$ ), surgical complications and need for additional surgical interventions. We also documented baseline characteristics including patient age, axial length, IOL power and prior peripheral iridotomy. Our patients were followed up over a two-year period. Success was defined as intraocular pressure of $<21 \mathrm{mmHg}$ with or without glaucoma medications.

The results are presented as mean $+/$ - standard deviation. Statistical analyses were performed with paired t-test and chi-squared test. A p value of $<0.05$ was considered statistically significant.

\section{Results}

Of 24 eyes in 19 patients, 5 had bilateral phacoemulsification with VSL. Two thirds were female, with a mean age of 75.7 years (range 6789 years). Ten eyes had prior peripheral iridotomy. Figure 3 shows the clinical characteristics of our patient population.

The mean pre-op and post-op IOP were 22.7 and $15.4 \mathrm{mmHg}$ respectively, with an average reduction of $7.3 \mathrm{mmHg}(\mathrm{p}=0.0003)$. The median number of glaucoma medications decreased from 2.1 presurgery to 1.8 post-surgery. No eyes required further IOP lowering procedures. Figure 4 shows the summary of pre-operative and postoperative parameters. A positive correlation was found between the preoperative IOP and last visit IOP drop ( $\mathrm{r}=0.94)$ (Figure 5).

The mean number of glaucoma medication dropped from $2.08 \pm$ 1.22 to $1.82 \pm 1.09$ ( $\mathrm{p}>0.05)$, with a positive correlation between pre-op and post-op eye drops $(\mathrm{r}=0.27)$ (Figure 6-8).

At final visit, success was achieved in $22 / 24$ (91.7\%) The most common intraoperative and postoperative complications were hyphema $(\mathrm{n}=1)$ and posterior capsule tear requiring anterior vitrectomy $(n=1)$. They all resolved with treatment.

\section{Conclusion}

P-VSL is an effective and safe procedure in controlling IOPs in patients with moderate to extensive PAS and could therefore delay or potentially prevent glaucoma filtration surgery. Further research needs to be done to better define its role in the management of PACG.

\section{Acknowledgements}

Paper presentation at the Congress of the European Society of

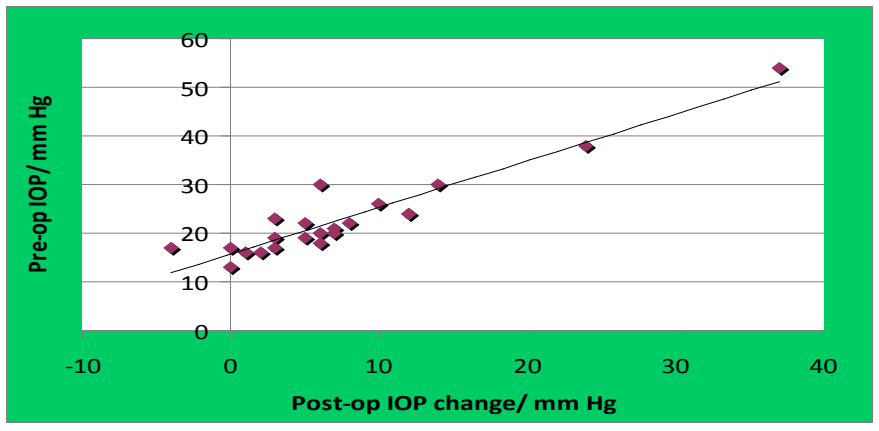

Figure 5. Positive correlation between post-op IOP change against pre-op IOP.

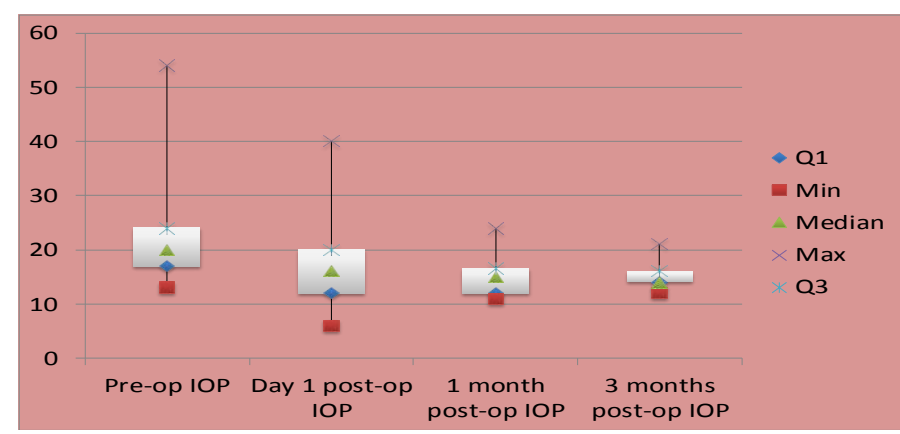

Figure 6. IOP profiles after p-VSL. 


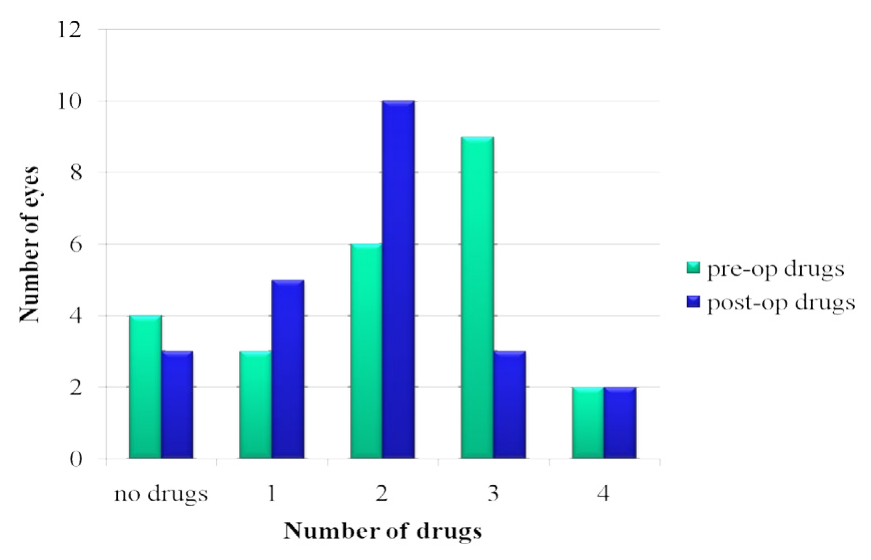

Figure 7. Number of glaucoma medications pre- and post-op.

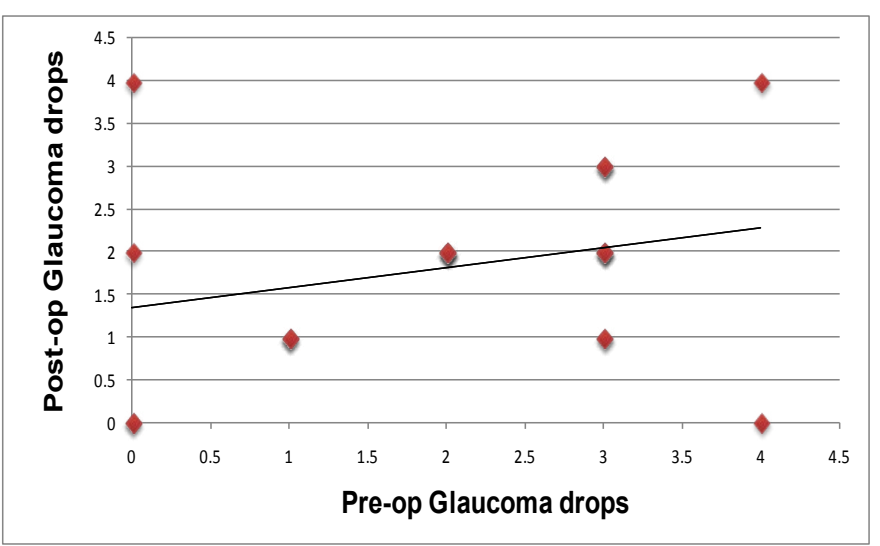

Figure 8. Correlation of post-op and pre-op glaucoma eye drops.
Ophthalmology, $11^{\text {th }}$ June 2013, Copenhagen, Denmark.

\section{References}

1. Quigley HA, Broman T (2006) The number of people with glaucoma worldwide in 2010 and 2020. Br J Ophthalmol 90: 262-267. [Crossref]

2. Ng WS, Ang GS, Azuara-Blanco A (2008) Primary angle closure glaucoma: a descriptive study in Scottish Caucasians. Clin Experiment Ophthalmol 36: 847-851. [Crossref]

3. Shams PN, Foster PJ (2012) Clinical outcomes after lens extraction for visually significant cataract in eyes with primary angle closure. $J$ Glaucoma 21: 545-550. [Crossref]

4. Kameda $\mathrm{T}$, Inoue $\mathrm{T}$, Inatani $\mathrm{M}$, Tanihara $\mathrm{H}$, Japanese Phaco-Goniosynechialysis Multicenter Study Group (2012) Long-term efficacy of goniosynechialysis combined with phacoemulsification for primary angle closure. Grafes Arch Clin Exp Ophthalmol 25: 825-830. [Crossref]

5. Morgan WH, Yu DY (2012) Surgical management of glaucoma: a review. Clin Experiment Ophthalmol 40: 388-399. [Crossref]

Copyright: (C2016 Jeganathan VSE. This is an open-access article distributed under the terms of the Creative Commons Attribution License, which permits unrestricted use, distribution, and reproduction in any medium, provided the original author and source are credited. 